\title{
Editorial
}

\section{Christine Busch}

In diesem Heft stelle ich Ihnen Beiträge zu einer Zielgruppe vor, die bisher wenig Beachtung in der Weiterbildung und in der Organisationsentwicklung findet. Es geht um die Zielgruppe der un- und angelernten Beschäftigten. Auch bei Arbeitswissenschaftlern und Interventionsforschern finden sich nur Wenige, die sich mit dieser Zielgruppe beschäftigen. Seit 2006 habe ich - dank des Bundesministeriums für Bildung und Forschung - die Chance, die Arbeitsbedingungen und die Gesundheit von Un- und Angelernten im Rahmen von zwei Verbundforschungsprojekten interdisziplinär zu beforschen und - und das ist das tatsächlich Bedeutsame - zielgruppenadäquate Interventionsprogramme zu entwickeln und zu evaluieren. Als wichtigste Ergebnisse der nunmehr ins siebte Jahr gehenden Bemühungen um verbesserte Weiterbildungs- und Gesundheitschancen für Un- und Angelernte zähle ich die publizierten Interventionsprogramme ReSuM (Ressourcen- und Stressmanagement für un- und angelernte Mitarbeiter durch Team- und Führungskräfteentwicklung) und ReSuDi (Ressourcen- und Stressmanagement für Un- und Angelernte bei hoher Diversität der Belegschaft), mit denen Weiterbildungs- und Präventionsanbieter in Betrieben Entwicklungs- und Gesundheitsförderung für Un- und Angelernte anbieten können.

Das Bundesministerium für Bildung und Forschung (BMBF) investiert nicht unerhebliche Gelder in die Entwicklung und Evaluation von Weiterbildungs- und Gesundheitsförderungskonzepten für sozial Benachteiligte, um - die in Deutschland sozial ungleich verteilten - Bildungs- und Gesundheitschancen für sozial Benachteiligte zu verbessern. Zudem wird vor dem Hintergrund der demografischen Entwicklung erkannt, dass Beschäftigte in un- und angelernten Tätigkeiten ein großes Potential darstellen, um den Fachkräftemangel vorzubeugen.

In den folgenden Beiträgen, die im Rahmen der vom BMBF geförderten Forschungsprojekte ReSuM und ReSuDi entstanden, stellen wir verschiedene Studien zu den Arbeitsbedingungen und zur Gesundheit von Un- und Anglernten dar. Angehörige der unteren sozialen Schichten tragen deutlich erhöhte Erkrankungs- und Sterberisiken im

Online publiziert: 19.04 .2013

(C) Springer Fachmedien Wiesbaden 2013

Dr. C. Busch $(\bowtie)$

Arbeits- und Organisationspsychologie,

Fachbereich Psychologie/Fakultät 4, Universität Hamburg,

Von-Melle-Park 11, 20146 Hamburg, Deutschland

E-Mail: cbusch@uni-hamburg.de 
Vergleich zu Personen mit höherem sozioökonomischen Status. Dabei zeigt die Forschung auf, dass psychosoziale Stressprozesse die größte Bedeutung für diese ungleich verteilten Risiken haben. Psychosoziale Belastungen resultieren im Besonderen aus den Arbeitsbedingungen.

Ein Beitrag beschäftigt sich daher mit den Arbeitsbedingungen und der Gesundheit von Beschäftigten in un- und angelernten Tätigkeiten. Un- und angelernte Beschäftigte arbeiten häufig in kulturell diversen Belegschaften. Sie haben im Vergleich zu qualifizierten Beschäftigten besonders belastende Arbeitsbedingungen. In diesem Beitrag wird der Stand der Forschung zur Arbeitssituation und zur gesundheitlichen Lage von Geringqualifizierten und Erwerbstätigen mit Migrationshintergrund dargestellt. Eine eigene Studie über die Arbeitsbedingungen und Gesundheit von geringqualifizierten Beschäftigten in kulturell diversen Belegschaften zeigt in Übereinstimmung mit der Literatur, dass die Beschäftigten belastende Arbeitssituationen haben und ein ungünstiges Gesundheitsverhalten zeigen. Die Arbeitssituation und Gesundheit von Beschäftigten mit und ohne Migrationshintergrund unterscheiden sich nicht. Migranten sind jedoch häufiger übergewichtig, rauchen seltener und haben ein besseres psychisches Wohlbefinden.

Ein weiterer Beitrag beschäftigt sich im Rahmen einer qualitativen Interviewstudie mit dem Gesundheitsverständnis von Beschäftigten in un- und angelernten Tätigkeiten. Die Studie zeigt, dass auch bei Un- und Angelernten ein komplexes Gesundheitsverständnis und die Kausalattribution typischer Arbeitsbedingungen auf negative Gesundheitsparameter vorherrschen. Agitation und Erschöpfung bzw. positive Stimmung und Energie waren dominante Reaktionen bei negativem bzw. positivem Befinden bei der Arbeit. Die Zuordnung der Interviewten zu Kulturclustern zeigte kulturelle Besonderheiten in subjektiven Gesundheitstheorien und Ursachen negativen Befindens. Diese Ergebnisse zeigen auf, wie in künftigen Studien Befindensbeeinträchtigungen und positives Wohlbefinden bei un- und angelernten, kulturell diversen Belegschaften zielgruppenadäquat erfasst werden können.

Ein dritter Beitrag untersucht den Einfluss der Teamarbeit auf die Gesundheit von unund angelernten Beschäftigten, die immerhin zu 50\% in Teams arbeiten. Die Forschung $\mathrm{zu}$ Teamarbeit und Gesundheit ist bisher vergleichsweise dünn. Dies gilt im Besonderen für Teams mit un- und angelernten Beschäftigten. In diesem Artikel wird aufbauend auf ein arbeitspsychologisches Ressourcen- und Stressmanagementmodell für Teams eine quantitative Studie mit Un- und Angelernten in Teamarbeit berichtet. Die Ergebnisse mittels Mehrebenenanalysen zeigen, dass die Qualität der Teamarbeit - die bei Un- und Angelernten vergleichsweise gering ist - bedeutsame Einflüsse auf den Stressprozess und die Gesundheit der Un- und Angelernten hat. Diese Einflüsse gehen über Einflüsse von soziodemografischen Variablen und individuellen Arbeitsressourcen und -stressoren hinaus. Daher sind Interventionen zur Entwicklung der Teamarbeit bei Un- und Angelernten notwendig. Das Programm ReSuM ist hierfür ein geeignetes Programm.

Ein vierter Beitrag beschäftigt sich empirisch mittels einer Interviewstudie mit dem gesundheitsbezogenen Führungsverhalten bei un- und angelernten, kulturell diversen Belegschaften. Un- und angelernte Beschäftigte in multikulturellen Belegschaften nannten als wichtige führungsbezogene Ressourcen Interesse und Präsenz der Führungskraft, wertschätzendes Feedback, die regelmäßige, rechtzeitige und ausreichende Weitergabe von Informationen und die Beteiligung der Mitarbeiter an Entscheidungen. Gleichzeitig 
gaben die befragten Mitarbeiter zu verstehen, dass es insbesondere an Wertschätzung und Partizipation mangele. Hier besteht besonderer Schulungsbedarf. Hier setzen die Programme ReSuM und ReSuDi mit ihren Führungskräfteschulungen für untere und mittlere Vorgesetzte an.

Ein fünfter Beitrag untersucht empirisch mittels einer qualitativen Interviewstudie die Lebensgestaltung und Work-Life Balance im Längsschnitt von un- und angelernten Frauen. Die befragten Frauen verfolgten ein traditionelles Geschlechtsrollenverständnis mit Fokus ihrer biografisch bedeutsamen Ziele auf den familiären Lebensbereich, unterschieden sich aber deutlich auf der Handlungsebene bzw. hinsichtlich der tatsächlich realisierten Lebensgestaltung. Es konnten fünf verschiedene Lebensgestaltungsformen identifiziert werden, in Abhängigkeit von erfahrenen Belastungen, Ressourcen und Bewältigungsstrategien. Im Programm ReSuM wird das Thema Ziele setzen und verfolgen für eine bessere Work-Life Balance aufgegriffen.

Ich hoffe, dass dieses Heft dazu beiträgt, dass Menschen in un- und angelernten Tätigkeiten von Personalentwicklern und Organisationsberatern sowie Weiterbildungs- und Präventionsanbietern stärker wahrgenommen werden. Betrieblichen Entscheidungsträgern können zielgruppenadäquate entwicklungs- und gesundheitsförderliche Interventionen für Un- und Angelernte, wie ReSuM oder ReSuDi, angeboten werden. Diese Maßnahmen sind bedeutsame Schritte auf dem Weg zu besseren Weiterbildungs- und Gesundheitschancen für Menschen in un- und angelernten Tätigkeiten.

Christine Busch 